\title{
Towards differential top-anti-top production at NNLO
}

\author{
Gabriel Abelof \\ Department of Physics \& Astronomy, Northwestern University, Evanston, IL 60208, USA \\ E-mail:gabriel.abelof@northwestern.edu
}

\section{Aude Gehrmann-De Ridder*}

Institute for Theoretical Physics, ETH, CH-8093 Zürich,

Physics Institute, University of Zürich, Winterthurerstrasse 190, CH-8057, Zürich

E-mail: gehra@itp.phys.ethz.ch

\section{Philipp Maierhöfer}

Physics Institute, University of Zürich, Winterthurerstrasse 190, CH-8057, Zürich

E-mail: philipp@physik.uzh.ch

\section{Stefano Pozzorini}

Physics Institute, University of Zürich, Winterthurerstrasse 190, CH-8057, Zürich

E-mail: pozzorin@physik.uzh.ch

We focus on the computation of NNLO corrections to the top-pair production cross section in the quark-antiquark channel. In order to capture the infrared behaviour of real-real and real-virtual matrix-elements, the NNLO antenna subtraction formalism extended to deal with processes having a massive fermion pair in the final state is used.

Loops and Legs in Quantum Field Theory

27 April 2014 - 02 May 2014

Weimar, Germany

\footnotetext{
* Speaker.
} 


\section{Introduction}

Top quark physics has become precision physics at the LHC. The ATLAS and CMS collaborations at CERN have reported first measurements of differential observables in top-quark pair production, such as the transverse momentum and rapidity of the $t \bar{t}$ system [1], its invariant mass [2], and the top quark transverse momentum [3]. Those measurements will allow a detailed probe of the top quark production mechanism. To reliably interpret these data, these precise measurements have to be matched onto equally accurate theoretical predictions. Those can be obtained by computing these hadron collider observables at the next-to-next-to leading order (NNLO) in perturbative QCD.

At NNLO, perturbative calculations of collider observables are typically carried out using parton-level event generators. These programs generate events for all parton-level subprocesses relevant to a given final state configuration up to NNLO accuracy and provide full kinematical information on an event-by-event basis.

Towards the computation of NNLO differential distributions for top pair production, in this talk, we present the status of the computation of the $\mathscr{O}\left(\alpha_{s}^{4}\right)$ corrections for top-pair production in the quark-antiquark channel. We focus on the following colour factor contributions: $N_{c}^{2}, N_{l} N_{c}$ and $N_{l} / N_{c}$, where $N_{l}$ and $N_{c}$ are respectively the number of light flavours and the number of colours.

An NNLO event generator for observables with $n$ final-state particles or jets involves three main building blocks: the two-loop corrections to the $n$-parton production process, denoted as double-virtual contributions $\mathrm{d} \sigma^{V V}$, the one-loop corrections to the $(n+1)$-parton production process, called real-virtual contribution $\mathrm{d} \sigma^{R V}$, and the tree-level $(n+2)$-parton double real contribution, $\mathrm{d} \sigma^{R R}$. These three building blocks involve infrared divergences that arise from the exchange or emission of soft and collinear partons and cancel only in their sum. In addition, the real-virtual and virtual-virtual contributions to hadron collider observables involve initial-state collinear singularities that must be absorbed into mass factorisation counter terms. Those are labelled as $\mathrm{d} \sigma^{M F, 1}$ and $\mathrm{d} \sigma^{M F, 2}$, respectively below.

The combination of subprocesses of different particle multiplicity and the consistent cancellation of the respective infrared singularities is one of the major challenges in the construction of NNLO parton-level event generators. In each subprocess, infrared singularities assume a different form: in the virtual corrections they are explicit, while in the real contributions they are implicit and become explicit only after phase space integration. To compute an observable beyond leading order, a regularization procedure is therefore required to extract and cancel the infrared singularities among different partonic channels before those can be implemented in the parton-level event generator. In the computations described here, we use the massive extension of the antenna formalism at NNLO to perform this task.

For computations with massive quarks, this formalism has been derived and applied so far for the evaluation of the following NNLO contributions. It has been used for the double real contributions to $t \bar{t}$ production for the pure fermionic processes [4] and for the gluon initiated process $g g \rightarrow t \bar{t} q \bar{q}$ [5]. More recently, this formalism has been derived to treat infrared divergences in realvirtual contributions for the leading colour contributions to $q \bar{q} \rightarrow t \bar{t}[6]$ and for the purely fermionic contributions (proportional to $N_{l}$.) in [7]. Results regarding these two partonic processes will be reported below. 
Employing a subtraction method, the NNLO partonic cross section for top-pair production in a given partonic channel (and proportional to a specific colour factor) has the general structure [8]

$$
\begin{aligned}
\mathrm{d} \hat{\sigma}_{\mathrm{NNLO}} & =\int_{\Phi_{4}}\left(\mathrm{~d} \hat{\sigma}_{\mathrm{NNLO}}^{\mathrm{RR}}-\mathrm{d} \hat{\sigma}_{\mathrm{NNLO}}^{\mathrm{S}}\right)+\int_{\Phi_{4}} \mathrm{~d} \hat{\sigma}_{\mathrm{NNLO}}^{\mathrm{S}} \\
& +\int_{\Phi_{3}}\left(\mathrm{~d} \hat{\sigma}_{\mathrm{NNLO}}^{\mathrm{RV}}-\mathrm{d} \hat{\sigma}_{\mathrm{NNLO}}^{\mathrm{VS}}\right)+\int_{\Phi_{3}} \mathrm{~d} \hat{\sigma}_{\mathrm{NNLO}}^{\mathrm{VS}}+\int_{\Phi_{3}} \mathrm{~d} \hat{\sigma}_{\mathrm{NNLO}}^{\mathrm{MF}, 1} \\
& +\int_{\Phi_{2}} \mathrm{~d} \hat{\sigma}_{\mathrm{NNLO}}^{\mathrm{VV}}+\int_{\Phi_{2}} \mathrm{~d} \hat{\sigma}_{\mathrm{NNLO}}^{\mathrm{MF}, 2}
\end{aligned}
$$

Two types of subtraction terms are introduced: $\mathrm{d} \hat{\sigma}_{\mathrm{NNLO}}^{\mathrm{S}}$ for the 4-parton final state, and $\mathrm{d} \hat{\sigma}_{\mathrm{NNLO}}^{\mathrm{VS}}$ for the 3-parton final state. The former approximates the behaviour of the double real contributions $\mathrm{d} \hat{\sigma}_{\mathrm{NNLO}}^{\mathrm{RR}}$ in their single and double unresolved limits, whereas the latter reproduces the single unresolved behaviour of the mixed real-virtual contributions $\mathrm{d} \hat{\sigma}_{\mathrm{NNLO}}^{\mathrm{RV}}$.

In the context of the antenna subtraction framework, we further decompose the double real subtraction term d $\hat{\sigma}_{\mathrm{NNLO}}^{\mathrm{S}}[9,10,11,12]$ such that,

$$
\begin{aligned}
\mathrm{d} \hat{\sigma}_{\mathrm{NNLO}} & =\int_{\Phi_{4}}\left[\mathrm{~d} \hat{\sigma}_{\mathrm{NNLO}}^{\mathrm{RR}}-\mathrm{d} \hat{\sigma}_{\mathrm{NNLO}}^{\mathrm{S}}\right] \\
& +\int_{\Phi_{3}}\left[\mathrm{~d} \hat{\sigma}_{\mathrm{NNLO}}^{\mathrm{RV}}-\mathrm{d} \hat{\sigma}_{\mathrm{NNLO}}^{\mathrm{T}}\right] \\
& +\int_{\Phi_{2}}\left[\mathrm{~d} \hat{\sigma}_{\mathrm{NNLO}}^{\mathrm{VV}}-\mathrm{d} \hat{\sigma}_{\mathrm{NNLO}}^{\mathrm{U}}\right]
\end{aligned}
$$

with

$$
\begin{aligned}
& \mathrm{d} \hat{\sigma}_{\mathrm{NNLO}}^{\mathrm{T}}=\mathrm{d} \hat{\sigma}_{\mathrm{NNLO}}^{\mathrm{VS}}-\int_{1} \mathrm{~d} \hat{\sigma}_{\mathrm{NNLO}}^{\mathrm{S}, 1}-\mathrm{d} \hat{\sigma}_{\mathrm{NNLO}}^{\mathrm{MF}, 1} \\
& \mathrm{~d} \hat{\sigma}_{\mathrm{NNLO}}^{\mathrm{U}}=-\int_{1} \mathrm{~d} \hat{\sigma}_{\mathrm{NNLO}}^{\mathrm{VS}}-\int_{2} \mathrm{~d} \hat{\sigma}_{\mathrm{NNLO}}^{\mathrm{S}, 2}-\mathrm{d} \hat{\sigma}_{\mathrm{NNLO}}^{\mathrm{MF}, 2}
\end{aligned}
$$

In [6], where we studied the leading colour contributions to top-pair production in the $q \bar{q}$ channel, it was shown that the subtraction terms constructed at the real-real and real-virtual level approximate the corresponding matrix-elements in all their infrared limits. Furthermore, an explicit and analytic pole cancellation occurs at the real-virtual level between explicit infrared poles of the one-loop matrix-elements and those from integrated double real subtraction terms.

In [7], where we compute the pure fermionic contributions to top-pair production in the $q \bar{q}$ channel, it is shown that the subtraction terms constructed at the real-virtual level approximate the real-virtual matrix-elements in all their infrared limits. Furthermore, combined with the integrated forms of the double real and real-virtual subtraction terms, we show that the explicit infrared poles cancel analytically at the real-virtual and virtual-virtual level respectively.

Besides the construction of subtraction terms, the calculation of the $2 \rightarrow 3$ one-loop amplitudes represent a non-trivial ingredient of the $2 \rightarrow 2$ NNLO calculation at hand. Those can in principle be provided using NLO tools. However, the application of NLO tools in the framework of NNLO calculations poses new and still poorly explored challenges. The integration of the (subtracted) one-loop contributions over the soft and collinear regions of phase space can lead to serious numerical instabilities. This issue has been addressed in [6]. It was shown that using the 
OPENLOOPS [13] one-loop generator in combination with the CuTTOOLS [14] reduction library, which allows to study the behaviour of one-loop matrix elements in the deep infrared regime using quadruple precision, those subtraction terms approximate the real-virtual matrix elements in all their singular limits. In addition, we found that these one-loop matrix elements building the realvirtual contributions are sufficiently stable numerically to perform a realistic NNLO calculation in double precision over almost the entire phase space regions. These statements will be detailed below. More precisely, this proceedings contribution is organised as follows: In Section 2 and 3 various detailed checks on the consistency and numerical stability of the double real and realvirtual subtractions related to $q \bar{q} \rightarrow t \bar{t}$ at leading colour are presented. Finally, Section 4 contains our conclusions and an outlook.

\section{Numerical tests of soft and collinear cancellations}

The double real and real-virtual contributions to heavy quark pair production in the $q \bar{q}$ channel at leading colour presented in [6] have been implemented in a Fortran code together with the corresponding subtraction terms $\mathrm{d} \hat{\sigma}_{q \bar{q}, \mathrm{NNLO}, N_{c}^{2}}^{\mathrm{S}}$ and $\mathrm{d} \hat{\sigma}_{q \bar{q}, \mathrm{NNLO}, N_{c}^{2}}^{\mathrm{T}}$. In this section we investigate how well these subtraction terms fulfil their purpose of approximating $\mathrm{d} \hat{\sigma}_{q \bar{q}, \mathrm{NNLO}, N_{c}^{2}}^{\mathrm{RR}}$ and $\mathrm{d} \hat{\sigma}_{q \bar{q}, \mathrm{NNLO}, N_{c}^{2}}^{\mathrm{RV}}$ in all unresolved limits.

For each singular region we used a series of phase-space samples generated with RAMBO [15] by requiring an increasingly small distance, parametrised in terms of appropriate parameters $x_{k}$, from the relevant singularity. In the next two subsections, we will quantify the level of the real-real and real-virtual cancellations as

$$
\delta_{\mathrm{RR}}=\left|\frac{\mathrm{d} \hat{\sigma}_{q \bar{q}, \mathrm{NNLO}, N_{c}^{2}}^{\mathrm{RR}}}{\mathrm{d} \hat{\sigma}_{q \bar{q}, \mathrm{NNLO}, N_{c}^{2}}^{\mathrm{S}}}-1\right|,
$$

and

$$
\delta_{\mathrm{RV}}=\left|\frac{\mathrm{d} \hat{\sigma}_{q \bar{q}, \mathrm{NNLO}, N_{c}^{2}}^{\mathrm{RV}}}{\mathrm{d} \hat{\sigma}_{q \bar{q}, \mathrm{NNLO}, N_{c}^{2}}^{\mathrm{T}}}-1\right|,
$$

respectively. To demonstrate the consistency and stability of the subtractions we will show that the $\delta_{\mathrm{RR}}$ and $\delta_{\mathrm{RV}}$ distributions converge to zero in specific $x_{k} \rightarrow 0$ limits. The results corresponding to those limits that are not presented here for conciseness can be found in [6]. On the righthand-side of (2.2) the consistent subtraction of explicit infrared singularities in the numerator and denominator is implicitly understood. Each of the employed samples consists of about $10^{4}$ points with $\sqrt{\hat{s}}=1 \mathrm{TeV}$ and $m_{Q}=174.3 \mathrm{GeV}$.

\subsection{Tests of the double real contributions}

In order to discuss infrared cancellations for the double real contribution $q \bar{q} \rightarrow Q \bar{Q} g g$ in leading colour approximation, we generated $2 \rightarrow 4$ phase space points near all possible single and double unresolved limits. The behaviour in the double soft limit is shown here. All other limits can be found in [6].

A double soft phase space point is characterised by the heavy quark pair taking nearly the full energy of the event, and therefore a suitable variable to control the proximity of the events to the 

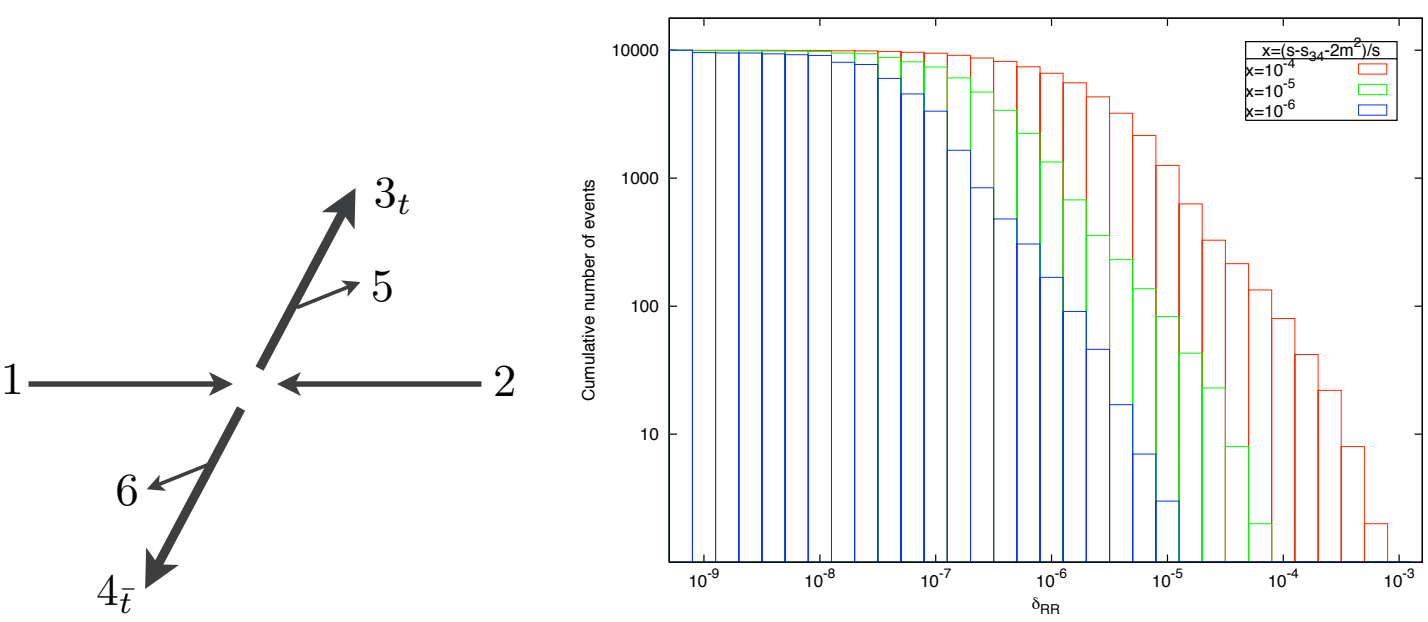

Figure 1: (a) Sketch of a double soft event. (b) Cumulative distributions of $\delta_{\mathrm{RR}}$ for $10^{4}$ double soft phase space points with three different values of $x$.

singular limit is $x=\left(s-s_{34}-2 m_{Q}^{2}\right) / s$. In Fig. 1.we show cumulative distributions of $\delta_{\mathrm{RR}}$ for three different values of $x$. Each bin contains the total number of points for which the relative difference between matrix element and subtraction term is larger than $\delta_{\mathrm{RR}}$. The good convergence of the subtraction terms to the double real contributions as the singularity is approached can be seen in the fact that the events accumulate more rapidly near $\delta_{R R}=0$ as the control variable $x$ is taken to be smaller.

\subsection{Tests of the real-virtual contributions}

In this subsection we study the cancellation between the real-virtual matrix elements and the corresponding subtraction terms. Only the soft limit of the gluon $p_{5} \rightarrow 0$ and the initial-final collinear limits $p_{i} \| p_{5}(i=1,2)$ must be considered.

The real-virtual cancellations provide a strong check both of the correctness of the subtraction terms and of the numerical stability of the OPENLOOPS amplitudes described in [6]. In the vicinity of the soft and collinear singularities matrix elements and subtraction terms are strongly enhanced, and the cancellation can amount to several digits. While this requires augmented numerical accuracy in the unsubtracted amplitudes, numerical instabilities related to Gram determinants can be strongly amplified in the vicinity of the singularities. It is thus crucial to prevent that the infrared cancellations are not spoiled by numerical instabilities of the amplitudes. The results presented in the following have been obtained with CUTTOoLs as a reduction back end of OpENLoops, using the quadruple-precision mode of CUTTOOLS as a rescue system for unstable points. Matrix elements are first evaluated in double precision and are reevaluated in quadruple precision if their estimated double-precision accuracy is less than 3 correct digits or smaller than the observed cancellation $\delta_{\mathrm{RV}}$ with the subtraction term as described in [6].

The left plot in Figure 2 shows the degree of cancellation $\delta_{\mathrm{RV}}$ in the soft region for samples of $10^{4}$ phase space points for several values of the control variable $x=\left(s-s_{34}-2 m_{Q}^{2}\right) / s$, which describes the softness of the phase space points. As the singularity is approached with smaller values 

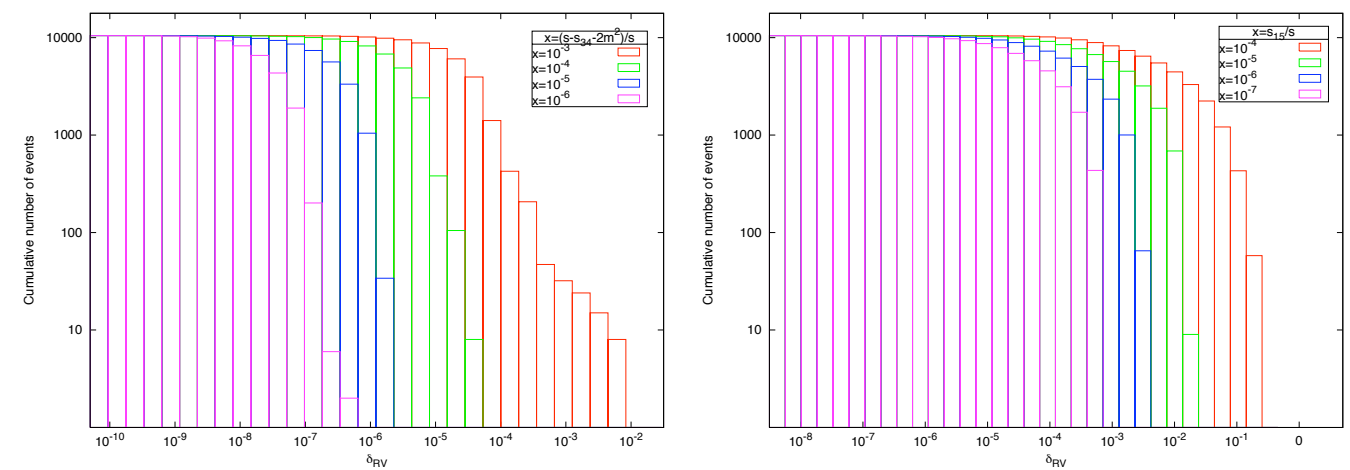

Figure 2: Distribution of $R$ for $10^{4}$ phase space points with three different values of $x$ for (left) soft and (right) collinear phase space configurations.

of $x$, the subtraction term $\mathrm{d} \hat{\sigma}_{q \bar{q}, \mathrm{NNLO}, N_{c}^{2}}^{\mathrm{T}}$ converges to the real-virtual corrections $\mathrm{d} \hat{\sigma}_{q \bar{q}, \mathrm{NNLO}, N_{c}^{2}}^{\mathrm{RV}}$ as expected. Similarly, the right plot in Figure 2 demonstrates the consistency of the cancellation in the collinear region, parametrised by the control variable $x=s_{15} / s$, where $p_{1}$ and $p_{5}$ are respectively the momenta of an incoming quark and an outgoing gluon.

For what concerns the numerical stability of the matrix elements in the collinear region, it turns out that for the depicted values of the control variable, double precision provides sufficient stability (in the sense of the criterion described above) for the vast majority of the phase space points.

\section{Stability of the integration over the three-particle phase space}

As a further test of the stability of the real-virtual matrix elements and of the related subtraction terms we have integrated the difference $\mathrm{d} \sigma_{q \bar{q}, \mathrm{NNLO}, N_{c}^{2}}^{\mathrm{RV}}-\mathrm{d} \sigma_{q \bar{q}, \mathrm{NNLO}, N_{c}^{2}}^{\mathrm{T}}$ inclusively over the threeparticle phase space employing a parton level event generator. In this integration, we impose a technical cut on the gluon $p_{T}$ using the technical cut $y_{\text {cut }}=p_{T}^{g} / \sqrt{\hat{s}}$, in such a way that no events are generated too close to the soft and collinear singularities. Naturally, since the entire phase space ought to be covered in the integration, $y_{\text {cut }}$ must be taken small. While the unsubtracted $\mathrm{d} \sigma^{\mathrm{RV}}$ contribution would lead to a logarithmic divergence in the limit $y_{\text {cut }} \rightarrow 0$, the subtraction term guarantees a smooth convergence at small $y_{\text {cut }}$. In practice the integral should reach a plateau for a sufficiently small value of the cut, $y_{\text {cut }}^{\max }$, i.e. for any $y_{\text {cut }}<y_{\text {cut }}^{\max }$ the integral of $\mathrm{d} \sigma^{\mathrm{RV}}-\mathrm{d} \sigma^{\mathrm{T}}$ should remain stable within Monte Carlo integration errors. This is clearly confirmed in Figure 3, where we plot the ratio

$$
\frac{\sigma_{q \bar{q}, \mathrm{NNLO}, N_{c}^{2}}^{\mathrm{RV}}-\sigma_{q \bar{q}, \mathrm{NNLO}, N_{c}^{2}}^{\mathrm{T}}}{\sigma_{q \bar{q}, L O}}
$$

for $p p \rightarrow t \bar{t}$ as a function of $y_{\text {cut }}$. For both the NNLO real-virtual subtracted contributions and the LO normalisation we used $\sqrt{s}=7 \mathrm{TeV}, m_{t}=174.3 \mathrm{GeV}$ and set the renormalisation and factorisation scales to $\mu_{R}=\mu_{F}=m_{t}$. We employed the MSTW2008nnlo90cl and MSTW20081o90cl PDF sets for the NNLO and the LO contributions respectively. The high stability of the integration results for values of $y_{\text {cut }}$ below $y_{\text {cut }}^{\max } \sim 10^{-3}$ provides solid evidence of the correctness of the real-virtual subtraction terms. Moreover, using OPENLOOPS in combination with CUTTOOLS, it 


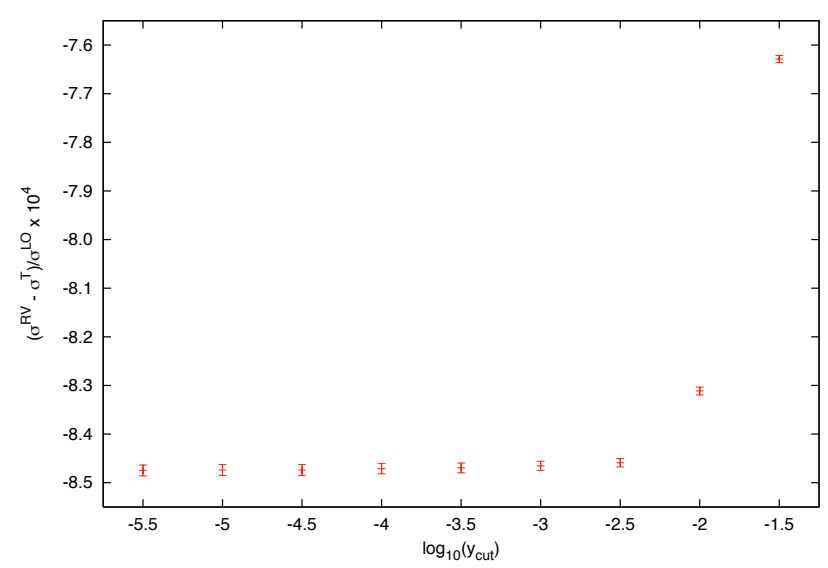

Figure 3: Inclusive phase space integral of $\mathrm{d} \sigma_{q \bar{q}, \mathrm{NNLO}, N_{c}^{2}}^{\mathrm{RV}}-\mathrm{d} \sigma_{q \bar{q}, \mathrm{NNLO}, N_{c}^{2}}^{\mathrm{T}}$ normalised to $\sigma_{q \bar{q}, L O}$ for different values of $y_{\text {cut }}$. The error bars correspond to the Monte Carlo uncertainty.

turns out that the stability plateau is reached before encountering significant instabilities in double precision. For $y_{\text {cut }}=10^{-3}\left(10^{-4}\right)$ we find that only 1 out of $10^{5}\left(10^{4}\right)$ events requires a quadruple precision reevaluation. This allows for a highly efficient evaluation of the real-virtual contributions based on double precision for the vast majority of the phase space points.

\section{Summary and outlook}

We presented results regarding the computation of double real and real-virtual NNLO contributions to hadronic $t \bar{t}$ production in the quark-antiquark channel at leading colour. This computation presented in [6] uses the massive extension of the antenna formalism at NNLO to capture the infrared behaviour of the real-real and real-virtual contributions. The correctness of the subtraction and its numerical stability have been demonstrated with detailed cancellation checks in section 2 .

To compute the one-loop $q \bar{q} \rightarrow t \bar{t} g$ real-virtual contributions we used OpEnLoops in combination with the CUTTOOLS implementation of OPP reduction. While the high CPU speed of OPENLOOPS represents an obvious attractive feature, numerical instabilities could represent a very serious issue for NNLO applications. To this end, using the quadrupole precision mode of the CUTTOOLS library, we performed detailed studies of the interplay between one-loop instabilities and infrared cancellations. Those have been presented in [6] and reported here too. On the one hand, it turns out that quadruple precision is essential to avoid excessive numerical instabilities in the deep infrared regime. On the other hand, we found that in the present context of NNLO calculations based on antenna subtraction, using a realistic infrared cut-off, one-loop amplitudes in double precision turn out to be sufficiently stable for the vast majority (more than 99.99\%) of the phase space points. The fact that quadruple precision can be avoided almost completely implies a drastic efficiency improvement for the integration of the real-virtual NNLO contributions. The results reported here constitute a major step towards a complete NNLO calculation based on antenna subtraction of top-pair production in the quark-antiquark channel. To complete the calculation of these NNLO 
corrections, the next task regards the computation of the two-parton contributions present at the virtual-virtual level for this partonic channel.

\section{Acknowledgments}

This research was supported by the Swiss National Science Foundation (SNF) under contracts PP00P2-139192, PP00P2-128552, and PBEZP2-145917 and in part by the European Commission through the 'LHCPhenoNet' (PITN-GA-2010-264564) and 'HiggsTools' (PITN-GA-2012316704) Initial Training Networks and the ERC Advanced Grant 'MC@ NNLO' (340983), which are hereby acknowledged.

\section{References}

[1] Georges Aad et al. Measurements of top quark pair relative differential cross-sections with ATLAS in $p p$ collisions at $\sqrt{s}=7$ TeV. Eur. Phys. J., C73:2261, 2013.

[2] CMS Collaboration. Measurement of differential top-quark pair production cross sections in the lepton+jets channel in pp collisions at $8 \mathrm{TeV}$. CMS-PAS-TOP-12-027, 2013.

[3] CMS Collaboration. Measurement of the differential top-quark pair production cross section in the dilepton channel in pp collisions at p s $=8 \mathrm{TeV}$. CMS-PAS-TOP-12-028, 2013.

[4] Gabriel Abelof and Aude Gehrmann-De Ridder. Double real radiation corrections to $t \bar{t}$ production at the LHC: the all-fermion processes. JHEP, 1204:076, 2012.

[5] Gabriel Abelof and Aude Gehrmann-De Ridder. Double real radiation corrections to $t \bar{t}$ production at the LHC: the $g g \rightarrow t \bar{t} q \bar{q}$ channel. JHEP, 1211:074, 2012.

[6] G. Abelof, A. Gehrmann-De Ridder, P. Maierhofer and S. Pozzorini. NNLO QCD corrections to top-antitop production in the $q \bar{q}$ channel. arXiv:1404.6493 [hep-ph], JHEP (to appear).

[7] G. Abelof, A. Gehrmann-De Ridder. In preparation.

[8] A. Gehrmann-De Ridder, T. Gehrmann, and Nigel Glover. Antenna subtraction at NNLO. JHEP, 0509:056, 2005.

[9] James Currie, Aude Gehrmann-De Ridder, E.W.N. Glover, and Joao Pires. NNLO QCD corrections to jet production at hadron colliders from gluon scattering. JHEP, 1401:110, 2014.

[10] James Currie, E.W.N. Glover, and Steven Wells. Infrared Structure at NNLO Using Antenna Subtraction. JHEP, 1304:066, 2013.

[11] Aude Gehrmann-De Ridder, Nigel Glover, and Joao Pires. Real-Virtual corrections for gluon scattering at NNLO. JHEP, 1202:141, 2012.

[12] Aude Gehrmann-De Ridder, Thomas Gehrmann, E.W.N. Glover, and Joao Pires. Double Virtual corrections for gluon scattering at NNLO. JHEP, 1302:026, 2013.

[13] Fabio Cascioli, Philipp Maierhofer, and Stefano Pozzorini. Scattering Amplitudes with Open Loops. Phys. Rev. Lett., 108:111601, 2012.

[14] Giovanni Ossola, Costas G. Papadopoulos, and Roberto Pittau. CutTools: A Program implementing the OPP reduction method to compute one-loop amplitudes. JHEP, 0803:042, 2008.

[15] R. Kleiss, W. James Stirling, and S.D. Ellis. A new Monte Carlo treatment of multiparticle phase space at high-energies. Comput. Phys. Commun., 40:359, 1986. 